\title{
Numerical computation of the Faradaic impedance of inlaid microdisk electrodes using a finite element method with anisotropic mesh adaptation
}

\author{
R. Michel ${ }^{1, \star}$, C. Montella ${ }^{1}$, C. Verdier ${ }^{2}$, J.-P. Diard $^{1}$
}

(1) Laboratoire d'Électrochimie et de Physicochimie des Matériaux et Interfaces, UMR 5631 CNRS +Grenoble-INP+UJF, Bât. PHELMA, 1130 Rue de la Piscine, B.P. 75, Domaine Universitaire, 38402 Saint Martin d'Hères, Cedex, France.

(2) Laboratoire de Spectrométrie Physique, UMR 5588 CNRS-UJF, Domaine Universitaire, 140 Avenue de la Physique, 38402 Saint Martin d'Hères, France.

* corresponding author.

Tel.: + 33-4-76826547; fax: + 33-4-76826630

e-mail: Richard.Michel@lepmi.grenoble-inp.fr

\begin{abstract}
The Faradaic impedance of a microdisk electrode inlaid in an insulating surface is revisited by numerical computation using a finite element method (FEM) with anisotropic mesh adaptation. New features of the numerical results, as compared to previous works, are analyzed. A first attractive feature is that the diffusion impedance relative to a microdisk electrode, evaluated at the equilibrium potential of the electrode, depends both on electron-transfer and mass-transport kinetics, in contrast with the usual behaviour of uniformly accessible electrodes. Next, the domain of validity of the Fleishmann and Pons semi-analytical formulation of diffusion impedance is determined. Finally, the characteristic of impedance graphs, which are the diffusion resistance, the characteristic frequency at the apex of the Nyquist diagram and the imaginary part of the diffusion impedance at this apex, are studied as functions of a dimensionless parameter that comparing the standard rate constant of electron transfer to the microelectrode diffusion constant. Closed form approximations are proposed for all quantities in order to help the analysis of experimental data.
\end{abstract}

Keyword: Impedance, Microelectrode, Microdisk, Simulation, Finite element method, Anisotropic mesh adaptation. 


\section{Introduction}

Despite the large literature dedicated to the theory of electrochemical impedance spectroscopy (EIS) [1-3] on the one hand and the theory of ultramicroelectrodes (UMEs) [4,5] with application to scanning electrochemical microscopy [6] on the other hand, only a few papers have dealt with the theoretical derivation of the impedance of a microdisk electrode inlaid in an insulating surface. The analysis presented by Fleischmann and Pons [7] opened up the use of microelectrodes to ac impedance measurements. They calculated the real and imaginary parts of the diffusion impedance from Bessel's function integrals. When achieving this work, the numerical evaluation of Fleischmann and Pons formulae was not an easy task. In order to make the impedance calculation easier, they presented their numerical data in the form of tabulated functions. Some additional information is available from the recent work by Navarro-Laboulais et al. [8]. These authors derived the theoretical formulation of the diffusion resistance that is the low-frequency limit of the diffusion impedance. They evaluated numerically the characteristic dimensionless frequency at the apex of the Nyquist impedance graph. An algorithm for calculation of the microdisk impedance was outlined by these authors for implementation in complex non-linear least-squares fitting (CNLS-Fit) programs.

An alternative approach for computing the impedance of microdisk electrodes is based on finite element analysis. The pioneering work on this topic was that of Ferrigno and Girault [9], which focused on the axisymmetric recessed microdisk geometry. As a limit, the inlaid disk electrode was recovered when the recess depth tends towards zero. A relatively good (qualitative) agreement was observed with the semi-analytical formulation of Fleischmann and Pons. However, this calculation was limited to the recessed microdisk geometry to avoid the presence of a singularity in the neighbourhood of the electrode edge where the boundary condition changes from the Dirichlet type (uniform concentration perturbation) on the disk to the homogeneous Neumann type (zero flux) on the insulator (see [10] for analysis of this kind of local singularities). The singularity refers to the concentration perturbation field which is not twice differentiable. Gabrielli 
et al. $[11,12]$ circumvented the above problem in recent articles relative to numerical simulation of the electrochemical impedance of an inlaid microdisk electrode using COMSOL Multiphysics (formerly FEMLAB) software. These authors investigated the influence of the disk radius and the total electrode radius (electroactive disk + insulating sheath) on the impedance diagram. Their theoretical predictions were compared to experimental data collected from a $10 \mu \mathrm{m}$ diameter Pt microdisk immersed in a $10 \mathrm{mM} \mathrm{K}{ }_{3} \mathrm{Fe}(\mathrm{CN})_{6}$ $+10 \mathrm{mM} \mathrm{K}_{4} \mathrm{Fe}(\mathrm{CN})_{6}+0.5 \mathrm{M} \mathrm{KCl}$ aqueous solution. The impedance was measured at the equilibrium potential of Pt electrode. Very good agreement was found for the impedance diagram simulated numerically using Fleischmann and Pons equations, as well as with the simulated FEM diagram. As discussed by Gabrielli et al. [11], Ferrigno and Girault [9] used a Dirichlet boundary condition at the disk/electrolyte interface (with the perturbation of interfacial concentration of electroactive species being directly controlled by the electrode potential perturbation), while Fleischmann and Pons [7] used a uniform non-homogeneous Neumann boundary condition (i.e. the distribution of the diffusional flux perturbation is assumed to be uniform over the disk surface). The more rigorous treatment proposed by Gabrielli et al. [11] makes use of the Fourier-Robin boundary condition that is the linearized formulation with respect to the electrode potential of the Butler-Volmer current-potential characteristic.

The aim of this work is an attempt to refine the numerical simulation of the impedance of inlaid microdisk electrodes using a finite element method (FEM) with anisotropic mesh adaptation. In this article, the computation procedure is employed for modeling the Faradaic impedance of microdisk electrodes at the equilibrium potential.

The results presented here are focused on the characteristic quantities available from impedance graphs, which are the diffusion resistance, the characteristic frequency observed at the apex of the Nyquist diagram, and the imaginary part of the impedance measured at this apex. Of course, the whole impedance diagram will be of major importance for its implementation in CNLS-Fit programs. However, the three quantities mentioned above are sufficient to compare the accuracy of the semi-analytical (Fleischmann and Pons) and numerical (FEM) procedures for computing the impedance of microdisk electrodes. 
First, the theory of microdisk electrodes is detailed in Section 2. The numerical method, including both the mesh adaptation strategy and some aspects of computer implementation, is presented in Section 3. Next, the influence of computational domain size on the accuracy of computed diffusion impedance is investigated in Section 4. The validity of Fleischmann and Pons formulae is checked in Section 5 by comparison with adaptative FEM computations. Finally, in Section 6, the characteristic elements from the diffusion impedance graph are analyzed with respect to the dimensionless number that compares the standard rate constant of electron transfer to the diffusion constant of microelectrodes.

\section{Theory}

\subsection{Geometry}

We consider a microdisk electrode inlaid in an insulating surface. The microdisk radius and the total electrode radius (electroactive disk + insulating sheath) are respectively denoted by $r_{\mathrm{e}}$ and $r_{\max }$. The geometry of the device is axisymmetric, so the calculation domain $\Omega$ is reduced to a $2-\mathrm{D}$ meridian section of the domain occupied by the electrolytic solution. This domain is sketched in Fig. 1 where $(r, z)$ denote the usual cylindrical coordinates. Its boundary $\Gamma$ decomposes into $\Gamma_{\mathrm{s}}$, the symmetry axis; $\Gamma_{\mathrm{e}}$, the electrode surface; $\Gamma_{\mathrm{i}}$, the insulator surface; and $\Gamma_{\mathrm{b}}$, the bulk electrolyte. These parts of the boundary $\Gamma$ are defined as follows:

$$
\begin{aligned}
& \Gamma_{\mathrm{s}}=\left\{(r, z) ; r=0 \text { and } 0<z<z_{\max }\right\} \\
& \Gamma_{\mathrm{e}}=\left\{(r, z) ; 0<r<r_{\mathrm{e}} \text { and } z=0\right\} \\
& \Gamma_{\mathrm{i}}=\left\{(r, z) ; r_{\mathrm{e}}<r<r_{\max } \text { and } z=0\right\} \\
& \Gamma_{\mathrm{b}}=\left\{(r, z) ;\left(r=r_{\max } \text { and } 0<z<z_{\max }\right) \text { or }\left(0 \leq r \leq r_{\max } \text { and } z=z_{\max }\right)\right\}(1 d
\end{aligned}
$$

$\Gamma_{\mathrm{b}}$ represents the part of the electrolyte which is located at a sufficient distance from the microdisk so that the influence of the electrochemical reaction (2) occurring at the disk surface can be neglected on $\Gamma_{\mathrm{b}}$. This assumption allows to impose the boundary condition 
of semi-infinite diffusion on $\Gamma_{\mathrm{b}}$ while, in a rigorous way, it should be imposed at an infinite distance from $\Gamma_{\mathrm{e}}$. The validity of this assumption mainly depends on the ratio $r_{\max } / r_{\mathrm{e}}$ as will be discussed in Section 4 .

\subsection{Phenomenology: electron-transfer and mass-transport pro- cesses}

A one-step electrochemical reaction occurs at the disk surface and involves a $n$-electrontransfer process (usually $n=1$ ) between two soluble species (O and $\mathrm{R}$ ) at the metal (e.g. Pt)/electrolyte interface $\Gamma_{\mathrm{e}}$ :

$$
\mathrm{O}+n \mathrm{e} \rightleftarrows \mathrm{R}
$$

The reaction rate is described by Butler-Volmer kinetics $[3,13]$ and, at the electrode potential $E$, it is expressed in the usual way by:

$$
v=k^{0}\left(\exp \left(-\alpha_{\mathrm{r}} \xi\right) c_{\mathrm{O}}-\exp \left(\alpha_{\mathrm{o}} \xi\right) c_{\mathrm{R}}\right) \text { with } \xi=(n F / R T)\left(E-E^{0^{\prime}}\right) \text { on } \Gamma_{\mathrm{e}}
$$

where $c_{\mathrm{X}}$ denotes the concentration of species $\mathrm{X}=\mathrm{O}, \mathrm{R}$, and the electron-transfer process is characterized by its standard rate constant $k^{0}$, the standard (formal) potential $E^{0^{\prime}}$, and the symmetry coefficients $\alpha_{\mathrm{o}}$ and $\alpha_{\mathrm{r}}$, with $\alpha_{\mathrm{o}}+\alpha_{\mathrm{r}}=1$, the other symbols having their usual meaning.

Due to the quiescent solution and the presence of a suitable supporting electrolyte, migration and convection effects on mass-transport processes can be neglected ${ }^{1}$, thus resulting in a pure diffusion process such that the flux-vector $\boldsymbol{J}_{\mathrm{X}}$ of species $\mathrm{X}=\mathrm{O}, \mathrm{R}$ is given by:

$$
\boldsymbol{J}_{\mathrm{x}}=-D_{\mathrm{x}} \nabla c_{\mathrm{x}} \quad \text { in } \quad \Omega
$$

where the diffusion coefficient $D_{\mathrm{x}}$ is a constant in the presence of supporting electrolyte,

\footnotetext{
${ }^{1}$ Strictly speaking, mass-transport of electroactive species by unforced convection should be taken into consideration using the model presented by Amatore et al. [14,15]. Nevertheless, these authors showed that the contribution of unforced convection can be neglected for an electrode radius $r_{\mathrm{e}}<25 \mu \mathrm{m}$. This condition is satisfied in our work.
} 
and $\nabla c_{\mathrm{x}}$ is the concentration gradient whose components in cylindrical coordinates are the partial derivatives, $\partial_{r} c_{\mathrm{x}}$ and $\partial_{z} c_{\mathrm{x}}$, of $c_{\mathrm{x}}$ with respect to $r$ and $z$ respectively.

\subsection{Structure of the Faradaic impedance}

\subsubsection{Steady-state problem}

The first step to analyse the Faradaic impedance is to calculate the steady-state regime corresponding to a static value $E_{\mathrm{S}}$ of the potential imposed to the electrode. This regime is characterized by the static concentrations $c_{\mathrm{S}, \mathrm{X}}$, for species $\mathrm{X}=\mathrm{O}, \mathrm{R}$, which are solutions of the following boundary value problem where the subscript 's' stands for steady-state conditions:

Given $E_{\mathrm{S}}$, find, for $\mathrm{X}=\mathrm{O}, \mathrm{R}, c_{\mathrm{S}, \mathrm{X}}:(r, z) \in \Omega \rightarrow c_{\mathrm{S}, \mathrm{X}}(r, z) \in \mathbb{R}$ such that ${ }^{2}:$

$$
\begin{array}{ccc}
-D_{\mathrm{x}} \Delta c_{\mathrm{S}, \mathrm{X}}=0 & \text { in } & \Omega \\
D_{\mathrm{x}} \boldsymbol{\nabla} c_{\mathrm{S}, \mathrm{x}} \cdot \boldsymbol{n}=\epsilon_{\mathrm{X}} v_{\mathrm{S}}\left(E_{\mathrm{S}}, c_{\mathrm{S}, \mathrm{O}}, c_{\mathrm{S}, \mathrm{R}}\right) & \text { on } & \Gamma_{\mathrm{e}} \\
D_{\mathrm{X}} \boldsymbol{\nabla} c_{\mathrm{S}, \mathrm{x}} \cdot \boldsymbol{n}=0 & \text { on } & \Gamma_{\mathrm{i}} \\
c_{\mathrm{S}, \mathrm{X}}=c_{\mathrm{x}}^{\mathrm{b}} & \text { on } & \Gamma_{\mathrm{b}}
\end{array}
$$

where $\epsilon_{\mathrm{O}}=-1, \epsilon_{\mathrm{R}}=+1, c_{\mathrm{X}}^{\mathrm{b}}$ denotes the bulk concentration of species X, $\boldsymbol{n}$ is the outward unit vector normal to $\Gamma$, and $\Delta$ is the Laplace operator whose cylindrical expression is given by $\Delta c_{\mathrm{S}, \mathrm{X}}=\partial_{r r}^{2} c_{\mathrm{S}, \mathrm{X}}+\frac{1}{r} \partial_{r} c_{\mathrm{S}, \mathrm{X}}+\partial_{z z}^{2} c_{\mathrm{S}, \mathrm{X}}$. The steady-state reaction rate $v_{\mathrm{S}}$ in Eq. (5b) comes from Eq. (3) where $E$ and $c_{\mathrm{X}}$ are respectively replaced by $E_{\mathrm{S}}$ and $c_{\mathrm{S}, \mathrm{x}}$. Once the problem (5) has been solved, the interfacial concentrations are known and the steady-state Faradaic current is obtained by integration along the radial direction $(r)$ as follows:

$$
I_{\mathrm{S}, \mathrm{f}}=-2 \pi n F \int_{\Gamma_{\mathrm{e}}} v_{\mathrm{S}} d s=-2 \pi n F \int_{0}^{r_{\mathrm{e}}} v_{\mathrm{S}}\left(E_{\mathrm{S}}, c_{\mathrm{S}, \mathrm{O}}, c_{\mathrm{S}, \mathrm{R}}\right) r d r
$$

\footnotetext{
${ }^{2}$ The boundary condition on $\Gamma_{\mathrm{S}}$ takes on the formulation, $r D_{\mathrm{X}} \boldsymbol{\nabla} c_{\mathrm{S}, \mathrm{x}} \cdot \boldsymbol{n}=0$, which results from the weak (variational) formulation of the boundary value problem used in the FEM framework. This is straightforwardly satisfied because of $r=0$ on $\Gamma_{\mathrm{s}}$. Hence, it is not necessary to write this boundary condition in Eq. (5). The same remark applies to the harmonic boundary value problem in Eq. (9).
} 
Note that the boundary condition (5d) is only an approximation of the semi-infinite diffusion boundary condition $c_{\mathrm{S}, \mathrm{X}}(r, z) \longrightarrow c_{\mathrm{x}}^{\mathrm{b}}$ when $r$ or $z \longrightarrow \infty$.

\subsubsection{Harmonic problem}

In the second step, a small harmonic perturbation of the electrode potential is imposed:

$$
E(t)=E_{\mathrm{S}}+E_{\mathrm{H}} \exp (j \omega t)
$$

where $j=\sqrt{-1}, \omega=2 \pi f$ is the angular frequency, $f$ is the frequency and $E_{\mathrm{H}}$ is the amplitude of perturbation. Under the above conditions, the permanent regime resulting from the potential perturbation leads to the following concentration fields:

$$
c_{\mathrm{X}}(r, z, t ; \omega)=c_{\mathrm{S}, \mathrm{X}}(r, z)+c_{\mathrm{H}, \mathrm{X}}(r, z ; \omega) \exp (j \omega t)
$$

The spatial part of the concentration fields perturbations $c_{\mathrm{H}, \mathrm{X}}(r, z ; \omega)$ are solutions of the following boundary value problem where the subscript ' $\mathrm{H}$ ' stands for the permanent harmonic regime and where the notation '; $\omega$ ' is used for highlighting the role of $\omega$ as a parameter:

Given $E_{\mathrm{S}}, E_{\mathrm{H}}$ and $\omega$, find, for $\mathrm{X}=\mathrm{O}, \mathrm{R}, c_{\mathrm{H}, \mathrm{X}}:(r, z) \in \Omega \rightarrow c_{\mathrm{H}, \mathrm{X}}(r, z) \in \mathbb{C}$ such that

$$
\begin{array}{ccc}
j \omega c_{\mathrm{H}, \mathrm{X}}-D_{\mathrm{X}} \Delta c_{\mathrm{H}, \mathrm{X}}=0 & \text { in } & \Omega \\
D_{\mathrm{X}} \boldsymbol{\nabla} c_{\mathrm{H}, \mathrm{X}} \cdot \boldsymbol{n}=\epsilon_{\mathrm{X}} v_{\mathrm{H}}\left(E_{\mathrm{S}}, c_{\mathrm{S}, \mathrm{O}}, c_{\mathrm{S}, \mathrm{R}}, E_{\mathrm{H}}, c_{\mathrm{H}, \mathrm{O}}, c_{\mathrm{H}, \mathrm{R}}\right) & \text { on } & \Gamma_{\mathrm{e}} \\
D_{\mathrm{X}} \boldsymbol{\nabla} c_{\mathrm{H}, \mathrm{X}} \cdot \boldsymbol{n}=0 & \text { on } & \Gamma_{\mathrm{i}} \\
c_{\mathrm{H}, \mathrm{X}}=0 & \text { on } & \Gamma_{\mathrm{b}}
\end{array}
$$

The harmonic perturbation of the reaction rate $v_{\mathrm{H}}$ results from linearization of the expression in Eq. (3) around the static polarization point $\left(E_{\mathrm{S}}, c_{\mathrm{S}, \mathrm{O}}, c_{\mathrm{S}, \mathrm{R}}\right)$. Using Eq. (7) for 
the potential and Eq. (8) for the concentration fields, we obtain:

$v_{\mathrm{H}}\left(E_{\mathrm{S}}, c_{\mathrm{S}, \mathrm{O}}, c_{\mathrm{S}, \mathrm{R}}, E_{\mathrm{H}}, c_{\mathrm{H}, \mathrm{O}}, c_{\mathrm{H}, \mathrm{R}}\right)=-k^{0}\left(\frac{n F}{R T} g_{\mathrm{S}} E_{\mathrm{H}}-\exp \left(-\alpha_{\mathrm{r}} \xi_{\mathrm{S}}\right) c_{\mathrm{H}, \mathrm{O}}+\exp \left(\alpha_{\mathrm{o}} \xi_{\mathrm{S}}\right) c_{\mathrm{H}, \mathrm{R}}\right)$

where

$$
g_{\mathrm{S}}=\alpha_{\mathrm{r}} \exp \left(-\alpha_{\mathrm{r}} \xi_{\mathrm{S}}\right) c_{\mathrm{S}, \mathrm{O}}+\alpha_{\mathrm{o}} \exp \left(\alpha_{\mathrm{o}} \xi_{\mathrm{S}}\right) c_{\mathrm{S}, \mathrm{R}} \quad \text { and } \quad \xi_{\mathrm{S}}=n F\left(E_{\mathrm{S}}-E^{0^{\prime}}\right) /(R T)
$$

\subsubsection{Harmonic perturbation of Faradaic current and Faradaic impedance}

Once the harmonic problem (9) is solved, the harmonic perturbations of interfacial concentrations are known and the permanent perturbation $I_{\mathrm{H}, \mathrm{f}}$ of the Faradaic current resulting from Eqs. (7) and (8) can be derived by integration along the radial direction $(r)$ as follows:

$$
I_{\mathrm{H}, \mathrm{f}}=-2 \pi n F \int_{\Gamma_{\mathrm{e}}} v_{\mathrm{H}} d s=-2 \pi n F \int_{0}^{r_{\mathrm{e}}} v_{\mathrm{H}}\left(E_{\mathrm{S}}, c_{\mathrm{S}, \mathrm{O}}, c_{\mathrm{S}, \mathrm{R}}, E_{\mathrm{H}}, c_{\mathrm{H}, \mathrm{O}}, c_{\mathrm{H}, \mathrm{R}}\right) r d r
$$

This expression is evaluated for each value of the angular frequency $\omega$. The ratio $I_{\mathrm{H}, \mathrm{f}} / E_{\mathrm{H}}$ defines the Faradaic admittance $Y_{\mathrm{f}}(\omega)$ as a function of $\omega$. Its formulation results directly from Eq. (10). It can be derived as:

$$
\begin{aligned}
Y_{\mathrm{f}}(\omega) & =G_{\mathrm{ct}}+Y_{\mathrm{dO}}(\omega)+Y_{\mathrm{dR}}(\omega) \\
G_{\mathrm{ct}} & =k^{0} \frac{n^{2} F^{2}}{R T} 2 \pi \int_{0}^{r_{\mathrm{e}}} g_{\mathrm{S}}(r, 0) r d r \\
Y_{\mathrm{dO}}(\omega) & =-k^{0} \frac{n F}{E_{\mathrm{H}}} \exp \left(-\alpha_{\mathrm{r}} \xi_{\mathrm{S}}\right) 2 \pi \int_{0}^{r_{\mathrm{e}}} c_{\mathrm{H}, \mathrm{O}}(r, 0 ; \omega) r d r \\
Y_{\mathrm{dR}}(\omega) & =k^{0} \frac{n F}{E_{\mathrm{H}}} \exp \left(\alpha_{\mathrm{o}} \xi_{\mathrm{S}}\right) 2 \pi \int_{0}^{r_{\mathrm{e}}} c_{\mathrm{H}, \mathrm{R}}(r, 0 ; \omega) r d r
\end{aligned}
$$

where $G_{\mathrm{ct}}$ is the electron-transfer conductance, and $Y_{\mathrm{dX}}$ denotes the concentration admittance relative to the species $\mathrm{X}=\mathrm{O}, \mathrm{R}$. Of course, the Faradaic impedance is obtained as

$$
Z_{\mathrm{f}}(\omega)=1 / Y_{\mathrm{f}}(\omega)
$$


Note that the electrochemical process and its mathematical formulation involve the correspondence $E_{\mathrm{H}} \longrightarrow I_{\mathrm{H}, \mathrm{f}}$. So it is the admittance which comes naturally from the above equations and not the impedance.

\section{Numerical resolution}

\subsection{Dimensionless formulation and limiting conditions}

\subsubsection{Dimensionless numbers}

It is easy to show that the solutions $c_{\mathrm{S}, \mathrm{O}}$ and $c_{\mathrm{S}, \mathrm{R}}$ of the steady-state boundary value problem (5) satisfy the relation:

$$
D_{\mathrm{O}} c_{\mathrm{S}, \mathrm{O}}(r, z)+D_{\mathrm{R}} c_{\mathrm{S}, \mathrm{R}}(r, z)=D_{\mathrm{O}} c_{\mathrm{O}}^{\mathrm{b}}+D_{\mathrm{R}} c_{\mathrm{R}}^{\mathrm{b}} \quad \text { for }(r, z) \in \Omega
$$

This property makes it possible to reduce the problem (5) to the determination of one concentration field only. Arbitrarily, we choose this concentration as $c_{\mathrm{S}, \mathrm{R}}$ and we use the diffusion coefficient and bulk concentration of species $\mathrm{R}$ as the reference quantities for the definition of the dimensionless variables $r^{\star}=r / r_{\mathrm{e}}, z^{\star}=z / r_{\mathrm{e}}, t^{\star}=t D_{\mathrm{R}} / r_{\mathrm{e}}^{2}, c_{\mathrm{S}, \mathrm{X}}^{\star}=c_{\mathrm{S}, \mathrm{X}} / c_{\mathrm{R}}^{\mathrm{b}}$

and $c_{\mathrm{H}, \mathrm{X}}^{\star}=c_{\mathrm{H}, \mathrm{X}} / c_{\mathrm{R}}^{\mathrm{b}}$ where superscript ' $\star$ ' indicates a dimensionless variable or operator. The dimensionless numbers resulting from this scaling are:

$$
\Lambda=\frac{k^{0} r_{\mathrm{e}}}{D_{\mathrm{R}}} \quad \text { and } \quad u=\frac{\omega r_{\mathrm{e}}^{2}}{D_{\mathrm{R}}}
$$

\subsubsection{Influence of $\Lambda:$ static conditions}

$\Lambda$ compares the standard rate constant of electron transfer to the diffusion constant of microelectrodes. It comes from the dimensionless form of the static boundary condition (5b) written for the species $\mathrm{X}=\mathrm{R}$ and combined together with Eq. (15) in order to eliminate $c_{\mathrm{S}, \mathrm{O}}$. The resulting equation is expressed by the following Fourier-Robin boundary 
condition written in dimensionless form:

$$
\nabla^{\star} c_{\mathrm{S}, \mathrm{R}}^{\star} \cdot \boldsymbol{n}^{\star}=\Lambda \alpha\left(\xi_{\mathrm{S}}\right)\left[c_{\mathrm{NS}, \mathrm{R}}^{\star}\left(\xi_{\mathrm{S}}\right)-c_{\mathrm{S}, \mathrm{R}}^{\star}\right] \quad \text { on } \quad \Gamma_{\mathrm{e}}^{\star}
$$

with:

$$
c_{\mathrm{NS}, \mathrm{R}}^{\star}\left(\xi_{\mathrm{S}}\right)=\left(\frac{D_{\mathrm{O}} c_{\mathrm{O}}^{\mathrm{b}}}{D_{\mathrm{R}} c_{\mathrm{R}}^{\mathrm{b}}}+1\right) \frac{1}{1+\frac{D_{\mathrm{O}}}{D_{\mathrm{R}}} \exp \left(\xi_{\mathrm{S}}\right)} \quad \text { and } \quad \alpha\left(\xi_{\mathrm{S}}\right)=\exp \left(\alpha_{\mathrm{o}} \xi_{\mathrm{S}}\right)+\frac{D_{\mathrm{R}}}{D_{\mathrm{O}}} \exp \left(-\alpha_{\mathrm{r}} \xi_{\mathrm{S}}\right)
$$

where $c_{\mathrm{NS}, \mathrm{R}}^{\star}\left(\xi_{\mathrm{S}}\right)$ stands for the dimensionless static interfacial concentration for Nernstian systems.

When $\Lambda \alpha\left(\xi_{\mathrm{s}}\right)$ is very large, the Fourier-Robin boundary condition (17a) leads to the Dirichlet boundary condition $c_{\mathrm{S}, \mathrm{R}}^{\star}=c_{\mathrm{NS}, \mathrm{R}}^{\star}\left(\xi_{\mathrm{S}}\right)$ over the disk surface $\Gamma_{\mathrm{e}}$. In contrast, at very low values of $\Lambda \alpha\left(\xi_{\mathrm{s}}\right)$, the Fourier-Robin boundary condition leads to a uniform nonhomogeneous Neumann boundary condition, i.e. assuming a uniform perturbation flux over the disk surface, except in a small neighbourhood of the electrode edge.

\subsubsection{Influence of $\Lambda$ : harmonic conditions}

When $D_{\mathrm{O}}=D_{\mathrm{R}}$, the perturbations of concentrations fields satisfy $c_{\mathrm{H}, \mathrm{O}}(r, z ; \omega)+c_{\mathrm{H}, \mathrm{R}}(r, z ; \omega)=$ 0 for $(r, z) \in \Omega$, which leads to the simplified formulation of Eqs. (9b) and (10):

$$
\nabla^{\star} c_{\mathrm{H}, \mathrm{R}}^{\star} \cdot \boldsymbol{n}^{\star}=-\Lambda \alpha\left(\xi_{\mathrm{S}}\right)\left[c_{\mathrm{NH}, \mathrm{R}}^{\star}+c_{\mathrm{H}, \mathrm{R}}^{\star}\right] \quad \text { on } \quad \Gamma_{\mathrm{e}}^{\star}
$$

with:

$$
c_{\mathrm{NH}, \mathrm{R}}^{\star}=\frac{\xi_{\mathrm{H}}}{\exp \left(-\alpha_{\mathrm{r}} \xi_{\mathrm{S}}\right)+\exp \left(\alpha_{\mathrm{o}} \xi_{\mathrm{S}}\right)} \frac{g_{\mathrm{S}}}{c_{\mathrm{R}}^{\mathrm{b}}}
$$

where $c_{\mathrm{NH}, \mathrm{R}}^{\star}$ stands for the dimensionless harmonic interfacial concentration perturbation for Nernstian systems and $\xi_{\mathrm{H}}=\frac{n F E_{\mathrm{H}}}{R T}$. Note that $g_{\mathrm{S}}$, obtained from Eq. (11), is constant over the electrode surface when the electrode impedance is calculated at the equilibrium potential. The situation would be much more intricate for impedance calculations performed away from the equilibrium potential. 
So the system kinetics is still governed by $\Lambda \alpha\left(\xi_{\mathrm{S}}\right)$ under harmonic conditions. Two limiting situations can be predicted. When $\Lambda \alpha\left(\xi_{\mathrm{s}}\right)$ is large, the harmonic perturbations of concentration fields tend to satisfy Dirichlet conditions at the disk/electrolyte interface like in the work of Ferrigno and Girault [9]. When $\Lambda \alpha\left(\xi_{\mathrm{s}}\right)$ is small, the harmonic perturbations of diffusional fluxes present approximately uniform values over the disk surface, and then, like in the work by Fleischmann and Pons [7], the Fourier-Robin boundary condition (18a) can be replaced by a uniform Neumann condition on $\Gamma_{\mathrm{e}}$. Both limiting conditions will be numerically verified in Section 6.2 .

At the opposite, when $D_{\mathrm{O}} \neq D_{\mathrm{R}}$, no simplification of harmonic equations is possible, so FEM computations should be performed with two concentration perturbation fields.

In the present work, we use the same approach as Gabrielli et al. [12], i.e. the FourierRobin boundary condition defined by Eq. (9b) without any approximation, except that the steady-state potential of the electrode is equal to its equilibrium potential, so $\xi_{\mathrm{s}}$ is given by the Nernst equation: $\xi_{\mathrm{S}}=\ln \left(c_{\mathrm{O}}^{\mathrm{b}} / c_{\mathrm{R}}^{\mathrm{b}}\right)$, therefore $\xi_{\mathrm{S}}=0$ when $c_{\mathrm{O}}^{\mathrm{b}}=c_{\mathrm{R}}^{\mathrm{b}}$.

\subsubsection{Influence of $u$}

The dimensionless angular frequency $u$ comes directly from the dimensionless form of Eq. $(9 a)$ :

$$
j u c_{\mathrm{H}, \mathrm{x}}^{\star}-\frac{D_{\mathrm{x}}}{D_{\mathrm{R}}} \Delta^{\star} c_{\mathrm{H}, \mathrm{x}}^{\star}=0 \quad \text { in } \quad \Omega^{\star}
$$

It compares the angular frequency $\omega$ with the reciprocal of diffusion time constant $r_{\mathrm{e}}^{2} / D_{\mathrm{R}}$. When $u$ is very large, it follows from the partial differential equation (19) that the concentration perturbations are vanishing in the electrolyte, except in the immediate neighbourhood of the disk surface (due to the boundary condition (18a)), so a boundary layer develops near $\Gamma_{\mathrm{e}}^{\star}$ at high frequencies. Conversely, at low frequencies, the concentration fields perturbations extend from the interface $\Gamma_{\mathrm{e}}^{\star}$ into the electrolyte until they vanish on $\Gamma_{\mathrm{b}}^{\star}$ 


\subsection{Mesh adaptation strategy}

Since the works of Nann and Heinze [16,17] and Harriman et al. [18-20], it has become widely accepted that, when performing simulations of electrochemical processes at microdisk electrodes, unstructured meshes must be used if a sufficiently accurate solution is to be obtained within a reasonable computing time. Adaptative finite element algorithms have been proposed and used for solving the mass-transport equations pertaining to the microdisk electrode geometry under steady-state [18-21], as well as timedependent $[17,22-24]$ conditions relative to the chronoamperometry, linear scan voltammetry and cyclic voltammetry methods. In the most recent articles, the main key factor is that the mesh refinement is under the control of an error estimator of the current [18-24].

Schematically, a mesh adaptation strategy associates two main stages. The first stage consists in the estimation of the error between the numerical approximation and the exact solution. The second stage consists in using this estimate to refine the mesh. In all previous works, the authors used a specific a posteriori estimation of the standard approximation error (see [25] for further details) and refined the mesh by subdivision of the current elements. Using this strategy, the error estimator should depend on the problem to be solved (i.e. mass-transport process, reaction mechanism and electrochemical method).

The mesh adaptation strategy used in this work is well documented in the article by Frey and Alauzet [26]. This method is based on the control of the interpolation error, which allows to control the approximation error and, hence, the accuracy of the numerical solution (see the contribution of Ciarlet [27] for a explanation of these concepts). On each mesh element, this interpolation error is locally bounded by a functional which depends on the tensor of second derivatives of the concentration perturbation (curvature tensor) and on the geometry of the element. From the eigenvectors and eigenvalues of this curvature tensor, computed at each vertex of the current mesh, it is possible to generate a new mesh for which the interpolation error is fixed to a specified value, and is constant over all elements and equidistributed in all directions. This adaptation process is performed by a complete remeshing without any reference to the current mesh. 
Fundamentally, this adaptation method allows to generate anisotropic meshes presenting highly stretched elements in arbitrary directions. This property is very useful to capture the behaviour of the concentration perturbations in the neighbourhood of the electrode edge (which introduces a singularity at all frequencies), as well as close to the microdisk surface (so-called boundary layer) where the concentration perturbation is essentially located in the high-frequency range. The whole procedure is repeated at each frequency, and, in particular the meshes, which lead to the specified interpolation error for two distinct frequencies, are distinct. A great advantage of the above strategy is that the mesh adaptation is of general purpose; it can be used irrespective of the electrochemical conditions: mass-transport process, reaction mechanism, cell geometry, electrochemical $\operatorname{method} .$.

An example of mesh generated by the anisotropic adaptation algorithm is presented in Fig. 2. The enlarged views highlight the refinement of the mesh near the singularity at the electrode edge. In addition, due to the high-frequency condition used in Fig. 2, the final mesh contains very stretched elements near the disk surface ("dark zone" in the figure). In this boundary layer, near the origin $\left(r / r_{\mathrm{e}} \approx 0\right)$, the radial dimension of mesh elements is 500 times larger than the axial dimension.

\subsection{Solvers, implementation and validation}

The FEM used in this work has been implemented in FreeFem++ developed by Hecht $[28,29]$, which is a powerful high-level language specially dedicated to weak formulation of boundary value problems for partial differential equations and to their approximations by finite element methods. Another particularly attractive feature of FreeFem++ is the fact that it provides a set of meshing tools allowing simple use of anisotropic mesh adaptation.

Finite element discretization of the partial differential equations (9) is performed using triangular elements and approximation by continuous piecewise quadratic polynomial on each triangle. This leads to a system of complex linear equations, which is solved by a Gaussian elimination algorithm stabilized by partial pivoting strategy. 
In order for the computation cost to remain reasonable, we also used the possibility of coupling FreeFem++ with the Davis's high performing solver UMFPack [30,31], the Goto's [32] and Whaley's [33] basic linear algebra subprograms (BLAS). The resulting computational environment allowed us to compute each Nyquist diagram in about half an hour on a Nocona processor running a 32-bits linux system. The whole computation, carried out at ninety different frequencies for each impedance graph, requires a little more than a thousand matrix factorizations of order varying from 2,500 to 1,600,000. Further details of the computation procedure can be found in Ref. [34].

The validity of the above strategy has been checked by comparison of the computed static concentration fields to the closed form solution derived by Crank and Furzeland [35] for Nerstian systems (i.e. setting $\Lambda \rightarrow \infty$ with our notation). A very good agreement has been observed. In addition, Fig. 3 clearly shows that the resulting approximation error on the concentration field is controlled by the interpolation error imposed by the mesh adaptation algorithm.

\section{Convergence vs. computational domain size}

\subsection{Nyquist plot}

Although it is the admittance that comes directly from Eqs. (13), as indicated above, the general use in electrochemistry is to plot impedance diagrams using the Nyquist representation, $-\operatorname{Im}\left(Z_{\mathrm{f}}\right)$ vs $\operatorname{Re}\left(Z_{\mathrm{f}}\right)$, with orthonormal axes. In addition, the influence of ohmic drop and double layer capacitance being neglected in this work, the electrode impedance reduces to the Faradaic impedance:

$$
Z_{\mathrm{f}}(u)=R_{\mathrm{ct}}+Z_{\mathrm{d}}(u)
$$


which is the sum of the diffusion impedance of soluble species $Z_{\mathrm{d}}(u)$ and the electrontransfer resistance:

$$
R_{\mathrm{ct}}=\lim _{u \longrightarrow \infty} Z_{\mathrm{f}}(u)=\frac{1}{G_{\mathrm{ct}}}
$$

where $G_{\mathrm{ct}}$ is given by Eq. (13b). The low-frequency limit of Faradaic impedance is the so-called polarization resistance $R_{\mathrm{p}}$ defined as:

$$
R_{\mathrm{p}}=\lim _{u \longrightarrow 0} Z_{\mathrm{f}}(u)=R_{\mathrm{ct}}+R_{\mathrm{d}}
$$

with the diffusion resistance being the limit:

$$
R_{\mathrm{d}}=\lim _{u \longrightarrow 0} Z_{\mathrm{d}}(u)
$$

Some Faradaic impedance graphs have been plotted in Fig. 4 for typical values of electrochemical parameters and different values of computational domain size, i.e. for $r_{\max } / r_{\mathrm{e}}$ ranging from 4 to 512. It should be noticed that, although all computations were performed with dimensionless numbers $\left(\xi_{\mathrm{S}}, \xi_{\mathrm{H}}, \Lambda, u \ldots\right)$ the impedance graphs and related quantities are presented here with their usual units in order to keep in mind the order of magnitude of the impedance of microdisk electrodes.

The higher $r_{\max } / r_{\mathrm{e}}$, the larger the frequency domain where the 'true' diffusion impedance relative to an inlaid microdisk electrode is computed with high accuracy.

An enlarged view of the low-frequency domain shows that the shape of the diagram, computed at small $r_{\max } / r_{\mathrm{e}}$ values, is quite different from that predicted by Fleischmann and Pons [7]. Indeed, the low frequency tail of the impedance graph corresponds to a small 'semicircle', rather than a straight line with slope (-1). This is in perfect agreement with the previous simulation by Gabrielli et al. [12]. However, a straight line with slope $(-1)$ is recovered for large computational domains, typically at $r_{\max } / r_{\mathrm{e}}=512$.

This can be quantified by investigating the convergence error of the Faradaic impedance 
with respect to the computation domain size. This error and its norm are defined by:

$$
\left\|\varepsilon_{\mathrm{f}, \mathrm{r}_{\max } / \mathrm{r}_{\mathrm{e}}}\right\|_{2}=\sqrt{\sum_{k}\left|\varepsilon_{\mathrm{f}, \mathrm{r}_{\max } / \mathrm{r}_{\mathrm{e}}}\left(u_{k}\right)\right|^{2}} \quad \text { with } \quad \varepsilon_{\mathrm{f}, \mathrm{r}_{\max } / \mathrm{r}_{\mathrm{e}}}(u)=\frac{Z_{\mathrm{f}, 512}(u)-Z_{\mathrm{f}, \mathrm{r}_{\max } / \mathrm{r}_{\mathrm{e}}}(u)}{Z_{\mathrm{f}, 4}(u)-Z_{\mathrm{f}, \mathrm{r}_{\max } / \mathrm{r}_{\mathrm{e}}}(u)}
$$

Here, $Z_{\mathrm{f}, \mathrm{r}_{\max } / \mathrm{r}_{\mathrm{e}}}(u)$ denotes the Faradaic impedance computed by FEM from Eqs (13) and (14) at the given value of $r_{\max } / r_{\mathrm{e}}$. The $u_{k}$ 's are the discrete values of dimensionless frequency in the different ranges defined in Fig. 5 where the decimal logarithm of the error norm has been plotted vs $r_{\max } / r_{\mathrm{e}}$ for the same parameters values than in Fig 4 . The four curves were obtained for impedance calculation carried out over different frequency ranges. For example, at $f \geq 10^{-2} \mathrm{~Hz}$, that is $u \geq 1.25 \times 10^{-2}$ in dimensionless notation, convergence is reached (i.e. a plateau is observed) as soon as $r_{\max } / r_{\mathrm{e}} \geq 40$. In contrast, $r_{\max } / r_{\mathrm{e}} \geq 200$ is required to compute accurately the impedance at frequency down to $10^{-4} \mathrm{~Hz}$. Finally, convergence is not yet reached, even at $r_{\max } / r_{\mathrm{e}}=256$, when the whole frequency domain $(u \geq 0)$ is taken into consideration. Note, however, that frequencies such that $f \leq 10^{-4} \mathrm{~Hz}$ are well beyond the range of experimentally accessible frequencies in electrochemistry, so the computational domain size $r_{\max } / r_{\mathrm{e}}=128$ will be used hereafter to plot impedance graphs.

\subsection{Polarization resistance}

We use the ratio $\left(R_{\mathrm{p}, \mathrm{r}_{\max } / \mathrm{r}_{\mathrm{e}}}-R_{\mathrm{p}, 4}\right) /\left(R_{\mathrm{p}, 512}-R_{\mathrm{p}, 4}\right)$ to define the normalized error on the polarization resistance. $R_{\mathrm{p}, \mathrm{r}_{\max } / \mathrm{r}_{\mathrm{e}}}$ is computed, at the given value of $r_{\max } / r_{\mathrm{e}}$, from Eqs. (13) and (14) after setting $\omega=0$ (i.e. $u=0$ ) in the harmonic boundary value problem (9). The normalized polarization resistance error has been plotted in Fig. 6 with respect to the computational domain size. An attractive feature is that convergence curves are the same for $k^{0}$ ranging from $10^{-5}$ to $10 \mathrm{~cm} \mathrm{~s}^{-1}$. The second feature is that convergence of $R_{\mathrm{p}}$ is reached within $5 \%$ relative error, typically for $r_{\max } / r_{\mathrm{e}} \geq 300$. 


\section{Validity domain for Fleischmann and Pons (FP) for- mulae}

FP formulae, reviewed in the Appendix A, are the references for computation of the impedance of microdisk electrodes. In previous works of Ferrigno and Girault [9], as well as Gabrielli et al. [12], good agreement with FP formulae was reported by the authors. Because of controlled computation accuracy in this work, we are now able to predict the validity domain of such formulae in terms of the parameter $\Lambda$ defined in Eq. (16).

It should be noted that the FP formulae were derived at the equilibrium potential assuming that $D_{\mathrm{O}}=D_{\mathrm{R}}=D$ and $c_{\mathrm{O}}^{\mathrm{b}}=c_{\mathrm{R}}^{\mathrm{b}}=c^{\mathrm{b}}$. The same assumptions are used here in FEM computations under harmonic conditions, so that $\xi_{\mathrm{S}}=0$ is satisfied in Eq. (18).

We compare $Z_{\mathrm{d}, \mathrm{FP}}(u)$, computed from the FP formulae (Eqs.(A.1) and (A.2) in the Appendix A), to $Z_{\mathrm{d}, \mathrm{r}_{\max } / \mathrm{r}_{\mathrm{e}}}(u)$, computed by self-adaptative FEM, thanks to the following measure of relative deviation:

$$
\left\|\varepsilon_{\mathrm{d}, \mathrm{r}_{\max } / \mathrm{r}_{\mathrm{e}}}\right\|_{2}=\sqrt{\sum_{k}\left|\varepsilon_{\mathrm{d}, \mathrm{r}_{\max } / \mathrm{r}_{\mathrm{e}}}\left(u_{k}\right)\right|^{2}} \quad \text { with } \quad \varepsilon_{\mathrm{d}, \mathrm{r} \max / \mathrm{r}_{\mathrm{e}}}(u)=\frac{Z_{\mathrm{d}, \mathrm{FP}}(u)-Z_{\mathrm{d}, \mathrm{r}_{\max } / \mathrm{r}_{\mathrm{e}}}(u)}{Z_{\mathrm{d}, \mathrm{FP}}(u)}
$$

The relative deviation has been plotted in Fig. 7 at different values of the standard rate constant of electron-transfer process. Convergence is reached for all $k^{0}$ values at increasing size of computational domain. In particular, the relative error converges very rapidly in the frequency domain $f \geq 10^{-3} \mathrm{~Hz}$, i.e. at $u \geq 1.25 \times 10^{-3}$.

Now, let us look at the plateau values. The relative error attains the asymptotic value $4 \times 10^{-3}$ when $k^{0} \leq 10^{-5} \mathrm{~cm} \mathrm{~s}^{-1}$, which indicates that the diffusion impedances computed from the FP formulae and adaptative FEM are very close to each others due to sluggish electron transfer kinetics at the microdisk surface. As soon as $k^{0}$ increases, the relative deviation also increases up to a constant value (not represented in the figure) at $k^{0} \geq$ $1 \mathrm{~cm} \mathrm{~s}^{-1}$. Indeed, the deviation between the two sets of impedance values is maximum for very fast charge-transfer kinetics. Hence, the FP formulae should not be employed to fit 
experimental impedance data measured from Nernstian systems.

The diffusion impedance computed using the self-adaptative FEM is also compared to the predictions from Fleischmann and Pons equations in Fig. 8 and 9 where the relative deviations of real and imaginary parts of the diffusion impedance have been plotted vs the decimal logarithm of frequency. For the intermediate value, $k^{0}=10^{-2} \mathrm{~cm} \mathrm{~s}^{-1}$, in Fig. 8, the deviation of $\operatorname{Im}\left(Z_{\mathrm{d}}\right)$ presents a bell-shaped curve, the maximum deviation (in absolute value) being close to $8 \%$ near the characteristic frequency of the impedance diagram. The relative deviation of $\operatorname{Re}\left(Z_{\mathrm{d}}\right)$ shows a more complex feature. The larger deviation, typically $5 \%$ in absolute value, is observed in the low-frequency range, while the two computation procedures are in good agreement with each other in the high-frequency domain (Warburg impedance).

In contrast, the relative deviation becomes very small due sluggish electron transfer (typically at $k^{0}=10^{-6} \mathrm{~cm} \mathrm{~s}^{-1}$ ). Scattering of numerical data in Fig. 9 is due to computational noise which is of the same order of magnitude than the relative deviation between the two computed impedances.

\section{Characteristic elements from the diffusion impedance graphs}

\subsection{Definitions}

The diffusion impedance (see Fig. 4) can be characterized by the diffusion resistance that is the low-frequency limit of $Z_{\mathrm{d}}$ in Eq. (23), the angular frequency $\omega_{\mathrm{c}}$ corresponding to the apex on the Nyquist diagram, and the imaginary part of $Z_{\mathrm{d}}$ measured at this apex.

The following dimensionless quantities will be used in what follows. First, $R_{\mathrm{d}}^{\star}$ denotes the dimensionless diffusion resistance:

$$
R_{\mathrm{d}}^{\star}=\frac{n^{2} F^{2} \pi r_{\mathrm{e}} D c^{\mathrm{b}}}{R T} R_{\mathrm{d}}
$$


where it is assumed that $D_{\mathrm{O}}=D_{\mathrm{R}}=D$ and $c_{\mathrm{O}}^{\mathrm{b}}=c_{\mathrm{R}}^{\mathrm{b}}=c^{\mathrm{b}}$. Next, $u_{\mathrm{c}}$ is the characteristic value of the dimensionless angular frequency at the apex of the Nyquist plot:

$$
u_{\mathrm{c}}=\omega_{\mathrm{c}} \frac{r_{\mathrm{e}}^{2}}{D}
$$

Finally, the dimensionless value of minus the imaginary part of $Z_{\mathrm{d}}$ measured at the apex is:

$$
\operatorname{Im} Z_{\mathrm{c}}^{\star}=-\frac{n^{2} F^{2} \pi r_{\mathrm{e}} D c^{\mathrm{b}}}{R T} \operatorname{Im} Z_{\mathrm{c}}
$$

\subsection{Influence of $\Lambda$; approximation formulae}

We now look for an approximation formula between each characteristic element and $\Lambda$.

The numerical procedure is as follows. Given any set of parameters values $\left(r_{\mathrm{e}}, k^{0}, D\right.$, $\left.c^{\mathrm{b}} \ldots\right)$, first, $R_{\mathrm{ct}}^{\star}$ and $R_{\mathrm{p}}^{\star}$ are computed using adaptative FEM under static conditions, and harmonic conditions with $u=0$, respectively. Next, both $u_{\mathrm{c}}$ and $\operatorname{Im} Z_{\mathrm{c}}^{\star}$ are obtained by coupling adaptative FEM computations with the accelerated golden section algorithm [36] and a smoothing procedure based on a parabolic fitting near the apex. Finally, $R_{\mathrm{d}}^{\star}, \operatorname{Im} Z_{\mathrm{c}}^{\star}$ and $u_{\mathrm{c}}$ are plotted as dots in Fig. 10 vs. the decimal logarithm of $\Lambda=\frac{k^{0} r_{\mathrm{e}}}{D}$. The ratio $I m Z_{\mathrm{c}}^{\star} / R_{\mathrm{d}}^{\star}$ is also plotted in this figure.

The four curves present interesting features not yet discussed in the electrochemical literature. First of all, the diffusion impedance relative to a microdisk electrode, evaluated at the equilibrium potential of the electrode, depends both on electron-transfer and masstransport kinetics, in contrast with the usual behaviour of uniformly accessible electrodes (see [3]). Indeed, the diffusion resistance is a function of $\Lambda=\frac{k^{0} r_{\mathrm{e}}}{D}$. The same remark applies to the imaginary part of the diffusion impedance at the apex of the Nyquist graph. Note, however, that the changes of $R_{\mathrm{d}}^{\star}$ and $\operatorname{Im} Z_{\mathrm{c}}^{\star}$ vs $\log (\Lambda)$ are not the same, i.e. the ratio $I m Z_{\mathrm{c}}^{\star} / R_{\mathrm{d}}^{\star}$ is not constant, so the impedance loop is more depressed at large values of $\Lambda$. Finally, a frequency shift can be predicted on the impedance diagram by looking at the change of $u_{\mathrm{c}}$ with respect to $\log (\Lambda)$ in Fig. 10 . 
Two horizontal asymptotes pertaining to small and large values of $\Lambda$ respectively, are clearly revealed in Fig. 10. First, considering very fast electron-transfer kinetics (large $\left.k^{0}\right)$, and/or a very large disk radius, and/or a very small diffusion coefficient, i.e. at very large values of $\Lambda$, the diffusion impedance is the same as the one obtained from the Dirichlet boundary condition initially employed by Ferrigno and Girault [9]. The limiting value for $R_{\mathrm{d}}^{\star}$ then is equal to $\pi / 2$ (see [34]).

In contrast, at very low values of $\Lambda$, due to a small $k^{0}$ and/or a very small disk radius, and/or a very large diffusion coefficient, the boundary condition (18a) is asymptotically the same as the uniform non-homogeneous Neumann boundary condition used by Fleischmann and Pons. This is clearly indicated in Fig. 10 by the upper limits of $R_{\mathrm{d}}^{\star}, \operatorname{Im} Z_{\mathrm{c}}^{\star}$ and $u_{\mathrm{c}}$ matching quasi-exactly the numerical values $R_{\mathrm{d}}^{\star}=16 /(3 \pi) \approx 1.698, \operatorname{Im} Z_{\mathrm{c}}^{\star} \approx 0.395$ and $u_{\mathrm{c}} \approx 2.440$ derived from the FP formulae in Appendix A.

Because of the asymptotic behaviours observed at small and large values of $\Lambda$, respectively, exponential functions are well suited to fit the numerical data in Fig. 10. Setting $\lambda=$ $\log (\Lambda)$ for the sake of simplification, and using a standard non-linear fitting procedure, the variations of $R_{\mathrm{d}}^{\star}, \operatorname{Im} Z_{\mathrm{c}}^{\star}$ and $u_{\mathrm{c}}$ with respect to $\lambda$ can be fitted accurately by the following function (among different possible fitting functions):

$$
f(\lambda)=a_{1}-\frac{a_{2}}{\left[1+b_{1} \exp \left(-b_{2} \lambda\right)\right]^{b_{3}}}
$$

where the constants $a_{1}$ and $a_{2}$ are directly obtained from the two horizontal asymptotes, while $b_{1}, b_{2}$ and $b_{3}$ are adjustable parameters. Their best-fitted values are given in Table 1 . The quality of the fit is illustrated by the solid lines plotted in Fig. 10, as well as the $\chi^{2}$ values reported in Table 1. It may be noticed that Eq. (29) is only a "representation model", so the best-fitted numerical coefficients have no particular physical meaning.

Looking at Fig. 10, it becomes very clear that the validity condition for the FP formulae is typically $\Lambda \leq 10^{-1}$, while the second limiting behaviour corresponding to Nernstian system kinetics is typically observed for $\Lambda \geq 10^{2}$. 


\subsection{Application to experimental data analysis}

Of course, a CNLS-Fit program should be used for accurate evaluation of the diffusion coefficient of electroactive species from EIS data measured at a microdisk electrode. However, a first evaluation of $D$ is possible, from experimental values of $R_{\mathrm{ct}}$ and $R_{\mathrm{d}}$, as follows. Assuming that $D_{\mathrm{O}}=D_{\mathrm{R}}=D$ and $c_{\mathrm{O}}^{\mathrm{b}}=c_{\mathrm{R}}^{\mathrm{b}}=c^{\mathrm{b}}$, the electron-transfer resistance evaluated at the equilibrium potential of a microdisk electrode is given by [7]:

$$
R_{\mathrm{ct}}=\frac{R T}{n^{2} F^{2} \pi r_{\mathrm{e}}^{2} k^{0} c^{\mathrm{b}}}
$$

Using Eqs. (16), (26), (29) and (30), we obtain:

$$
\frac{R_{\mathrm{d}}}{R_{\mathrm{ct}}}=\Lambda f(\lambda)
$$

Given experimental values for the disk radius and the resistances $R_{\mathrm{d}}$ and $R_{\mathrm{ct}}$, Eqs. (29) and (31), employed together with the relevant numerical coefficients in the first entry of Table 1, result in an implicit equation with respect to $\Lambda=k^{0} r_{\mathrm{e}} / D$.

This equation can be readily solved for $k^{0} / D$, e.g. using the 'FindRoot' command of Mathematica [37] or the one-dimensional Root-Finding algorithm of the Gnu scientific library [38], while the standard rate constant of electron transfer can be directly obtained from the electron-transfer resistance in Eq. (30), so the diffusion coefficient can be readily evaluated.

By way of illustration, let us consider the EIS data collected by Gabrielli et al. [12] from a $10 \mu \mathrm{m}$ diameter Pt microdisk immersed in a $10 \mathrm{mM} \mathrm{K}_{3} \mathrm{Fe}(\mathrm{CN})_{6}+10 \mathrm{mM} \mathrm{K} \mathrm{Fe}_{4}(\mathrm{CN})_{6}+$ $0.5 \mathrm{M} \mathrm{KCl}$ aqueous solution. The impedance was measured at the equilibrium potential of Pt electrode. The values, $R_{\mathrm{ct}} \approx 0.85 \mathrm{M} \Omega$ and $R_{\mathrm{d}} \approx 4.15 \mathrm{M} \Omega$, can be extracted from the impedance spectra. Using the above calculation procedure, we obtain $k^{0}=4.0 \times$ $10^{-2} \mathrm{~cm} \mathrm{~s}^{-1}$ and $D=6.7 \times 10^{-6} \mathrm{~cm}^{2} \mathrm{~s}^{-1}$. Such values can be favorably compared to those values obtained by Gabrielli et al. using a CNLS-Fit program (Simplex algorithm), i.e. 
$k^{0}=3.6 \times 10^{-2} \mathrm{~cm} \mathrm{~s}^{-1}$ and $D=6.6 \times 10^{-6} \mathrm{~cm}^{2} \mathrm{~s}^{-1}$.

\section{Conclusions}

The major points of this article are the following.

- A finite element method with anisotropic mesh adaptation has been used for the first time to compute electrochemical impedance data, in the case of a microdisk electrode inlaid in an insulating surface.

- The mesh adaptation strategy used in this work is of general purpose. It allows the use of anisotropic meshes and provides a numerical solution with uniform accuracy (fixed by the user) over each mesh element. This strategy can be employed irrespective of the electrochemical conditions. For example, the mesh adaptation strategy is used both for solving the steady-state boundary value problem and the harmonic boundary value problem.

- The numerical method used in this work has been implemented in FreeFem++ which is a powerful high-level language specially dedicated to weak formulation of boundary value problems for partial differential equations and to their approximations by finite element methods. FreeFem++ provides a set of meshing tools allowing a simple use of anisotropic mesh adaptation and can be coupled with the Davis's high performing solver UMFPack and specialized algebra library.

- The size of the finite element calculation domain is a crucial parameter for computing the Faradaic impedance of microdisk electrodes. A large ratio of the total radius to the disk radius, typically $r_{\max } / r_{\mathrm{e}} \geq 128$, is required to attain high accuracy in the frequency range $f \geq 10^{-3} \mathrm{~Hz}$.

- We have shown that the diffusion impedance, computed by self-adaptative FEM at the equilibrium potential of the electrode, depends on the dimensionless parameter 
$k^{0} r_{\mathrm{e}} / D$. This behaviour strongly differs from that predicted for uniformly accessible electrodes [3].

- The impedance of a microdisk electrode depends, of course, on the boundary condition at the metal surface. In this work, we used the Fourier-Robin boundary condition, which is the linearized formulation of the Butler-Volmer current-potential characteristic.

- The validity of the semi-analytical formulation of $Z_{\mathrm{d}, \mathrm{FP}}(u)$, derived in the pioneering work by Fleischmann and Pons [7], has been discussed for impedance computations carried out at the equilibrium potential of the electrode. From our finite element simulations, we came to the conclusion that the semi-analytical formulation is only valid at low values of $k^{0} r_{\mathrm{e}} / D$, typically at $k^{0} r_{\mathrm{e}} / D \leq 10^{-1}$. In contrast, at intermediate or large values of $k^{0} r_{\mathrm{e}} / D$, deviation of $Z_{\mathrm{d}}$ from Fleischmann and Pons formulae was predicted. The maximum deviation is of the order of $8-15 \%$ in terms of the diffusion resistance, the characteristic frequency at the apex of the Nyquist diagram, and the value of $-\operatorname{Im}\left(Z_{\mathrm{d}}\right)$ at this apex. A frequency shift is also predicted on the impedance diagram, which depends both on frequency and the $k^{0} r_{\mathrm{e}} / D$ value.

- The results presented here are essentially focused on the characteristic quantities available from impedance graphs, which are the diffusion resistance, the characteristic frequency at the apex of the Nyquist impedance graph, and the imaginary part of the impedance measured at this apex. Eqs. (26)-(28) are the theoretical formulations for these quantities, with $R_{\mathrm{d}}^{\star}, \operatorname{Im} Z_{\mathrm{c}}^{\star}$ and $u_{\mathrm{c}}$ being evaluated numerically by adaptative FEM.

- The variations of $R_{\mathrm{d}}^{\star}, \operatorname{Im} Z_{\mathrm{c}}^{\star}$ and $u_{\mathrm{c}}$ with respect to $\log \left(k^{0} r_{\mathrm{e}} / D\right)$ have been fitted accurately by the function in Eq. (29), employed together with the numerical coefficients in Table 1, in order to make the evaluation of such characteristic quantities easier whatever the values of the electrochemical system parameters.

- We have shown that the above quantities can be used for the evaluation of the 
diffusion coefficient of soluble species from experimental EIS data at a microdisk electrode.

\section{References}

[1] C. Gabrielli, in I. Rubinstein (Ed.), Physical Electrochemistry. Principles, methods, and applications, Marcel Dekker, New York, 1995.

[2] J.R. MacDonald, W.B. Johnson, in J. R. Macdonald and E. Barsoukov, (Ed.), Impedance Spectroscopy. Theory, Experiment, and Applications, John Wiley \& Sons, Inc., Hoboken, New Jersey, 2005.

[3] J.-P. Diard, B. Le Gorrec, C. Montella, Cinétique électrochimique, Hermann, Paris, 1996.

[4] M. Fleischmann, S. Pons, D.R. Rolinson, P.P. Schmidt, Ultramicroelectrodes, Datatech System Inc., Morganton, NC, 1987.

[5] C. Amatore, in I. Rubinstein (Ed.), Physical Electrochemistry. Principles, methods, and applications, Marcel Dekker, New York, 1995.

[6] A.J. Bard, F.-R.F. Fan, M.V. Mirkin, in I. Rubinstein (Ed.), Physical Electrochemistry. Principles, methods, and applications, Marcel Dekker, New York, 1995.

[7] M. Fleischmann, S. Pons, J. Electroanal. Chem. 250 (1988) 277.

[8] J. Navarro-Laboulais, J.J. Garcia-Jareño, F. Vicente, J. Electroanal. Chem. 536 (2002) 11.

[9] R. Ferrigno, H.H. Girault, J. Electroanal. Chem. 492 (2000) 1.

[10] P. Grisvard, Singularities in Boundary value Problems, Research Notes in Applied Mathematics, Vol. 22, Masson, Paris, 1992.

[11] C. Gabrielli, F. Huet, M. Keddam, P. Rousseau, V. Vivier, J. Phys. Chem. B 108 (2004) 11620. 
[12] C. Gabrielli, M. Keddam, N. Portail, P. Rousseau, H. Takenouti, V. Vivier, J. Phys. Chem. B 110 (2006) 20478.

[13] A.J. Bard, L.R. Faulkner, Electrochemical methods. Fundamentals and applications, John Wiley \& Sons, Inc., New York, 2001.

[14] C. Amatore, S Szunerits, L. Thouin, J.-S. Warkocz, J. Electroanal. Chem. 500 (2001) 62.

[15] C. Amatore, C. Pebay, L. Thouin, A. Wang, Electrochem. Commun. 11 (2009) 1269.

[16] T. Nann, J. Heinze, Electrochem. Commun. 1 (1999) 289.

[17] T. Nann, J. Heinze, Electrochim. Acta 48 (2003) 3975.

[18] K. Harriman, D.J. Gavaghan, P. Houston, E. Süli, Electrochem. Commun. 2 (2000) 150.

[19] K. Harriman, D.J. Gavaghan, P. Houston, E. Süli, Electrochem. Commun. 2 (2000) 157.

[20] K. Harriman, D.J. Gavaghan, P. Houston, E. Süli, Electrochem. Commun. 2 (2000) 163.

[21] S.C.B. Abercrombie, G. Denuault, Electrochem. Commun. 5 (2003) 647.

[22] K. Harriman, D.J. Gavaghan, E. Süli, J. Electroanal. Chem. 569 (2004) 35.

[23] K. Harriman, D.J. Gavaghan, E. Süli, J. Electroanal. Chem. 573 (2004) 169.

[24] É. Mahé, Electrochim. Acta 52 (2007) 5018.

[25] M. Ainsworth, J.T. Oden, A Posteriori Error Estimation in Finite Element Analysis, John Wiley \& Sons, Inc., New York, 2000.

[26] P. Frey, F. Alauzet, Comput. Methods Appl. Mech. Engrg. 194 (2005) 5068.

[27] P.G. Ciarlet, in P. G. Ciarlet and J. L. Lions, (Ed.), Handbook of Numerical Analysis, volume II, North-Holland, Amsterdam, 1991. 
[28] F. Hecht, Langage FreeFem++. http://www.freefem.org.

[29] F. Hecht, Mod. Math. Anal. Num. 36 (2002) 809.

[30] T.A. Davis, UMFPack, http://www.cise.ufl.edu/research/sparse/umfpack.

[31] T.A. Davis, Direct Methods for Sparse Linear Systems, SIAM Press, Philadelphia, 2006.

[32] K. Goto, Library GotoBLAS, http://www.tacc.utexas.edu/resources/software.

[33] R.C. Whalley, Library ATLAS, http://math-atlas.sourceforge.net.

[34] R. Michel, PhD thesis, Institut National Polytechnique, Grenoble, 2009.

[35] J. Crank, R.M. Furzeland, J. Inst. Math. Appl. 20 (1997) 355.

[36] R.P. Brent, Algorithms for Minimization without Derivatives, Prentice-Hall, Englewood Cliffs, 1973.

[37] S. Wolfram, The Mathematica book, sixth edition, Wolfram Media \& Cambridge University Press, Champaign-USA, Cambridge-UK, 2007.

[38] Gnu Project, Gnu Scientific Library, http://www.gnu.org/software/gls.

[39] G.N. Watson, A Treatise on the Theory of Bessel functions, Cambridge University Press, Cambridge, 1995.

[40] Z.A. Rotenberg, A.V. Drobinskii, V.P. Lukovtsev, N.S. Khozyainova, Elektrokhimiya 36 (2000) 996.

\section{A Faradaic impedance derived from Fleischmann and Pons formulae}

In the seminal work by Fleischmann and Pons [7] the impedance of a microdisk electrode was calculated assuming that both oxidized and reduced species have the same diffusion 
coefficient $\left(D_{\mathrm{O}}=D_{\mathrm{R}}=D\right)$ and the same bulk concentration $\left(c_{\mathrm{O}}^{\mathrm{b}}=c_{\mathrm{R}}^{\mathrm{b}}=c^{\mathrm{b}}\right)$. In addition, Ohmic potential drop and double-layer effects were disregarded, so the electrode impedance reduces to the Faradaic impedance. The major assumptions are, firstly, that the impedance is calculated at the equilibrium potential of the electrode, and, secondly, that the diffusion flux perturbation is supposed uniform over the microdisk surface. The resulting discontinuity in the boundary condition, which changes from the uniform nonhomogeneous Neumann boundary condition (uniform flux perturbation) on the microdisk to the homogeneous Neumann boundary condition (zero flux) on the insulator, was taken into account thanks to the Weber-Schafheitlin integral [39], so-called "discontinuous definite Bessel integrals" in Ref. [7].

The electron-transfer resistance relative to the electrochemical reaction in Eq. (2), evaluated at the equilibrium potential of the disk electrode with the same bulk concentration of oxidized and reduced species in the electrolytic solution, is given by Eq. (30). The Faradaic impedance relative to the electrochemical reaction in Eq. (2), can be written as the sum of $R_{\mathrm{ct}}$ and the diffusion impedance of soluble species $Z_{\mathrm{d}}(u)$ according to Eq. (20). Starting from Fleischmann and Pons formulae [7], the real part (Re) and the imaginary part $(\mathrm{Im})$ of the diffusion impedance can be written respectively as:

$$
\operatorname{Re}\left(Z_{\mathrm{d}, \mathrm{FP}}(u)\right)=\frac{4 R T}{n^{2} F^{2} \pi r_{\mathrm{e}} D c^{\mathrm{b}}} \frac{\Phi_{4}(u)}{\sqrt{u}}
$$

and:

$$
\operatorname{Im}\left(Z_{\mathrm{d}, \mathrm{FP}}(u)\right)=-\frac{4 R T}{n^{2} F^{2} \pi r_{\mathrm{e}} D c^{\mathrm{b}}} \frac{\Phi_{5}(u)}{\sqrt{u}}
$$

with the functions $\Phi_{4}(u)$ and $\Phi_{5}(u)$ being defined as the following integrals:

$$
\Phi_{4}(u)=\int_{0}^{\infty}\left[\mathrm{J}_{1}(x \sqrt{u})\right]^{2} \frac{\cos (\theta / 2)}{x\left(1+x^{4}\right)^{1 / 4}} d x
$$

and:

$$
\Phi_{5}(u)=\int_{0}^{\infty}\left[\mathrm{J}_{1}(x \sqrt{u})\right]^{2} \frac{\sin (\theta / 2)}{x\left(1+x^{4}\right)^{1 / 4}} d x
$$


where $J_{1}(y)$ is the Bessel function of the first kind and first order, with the real argument $y=x \sqrt{u}$, and $\theta=\arctan \left(1 / x^{2}\right)$.

First, using a computer algebra system like Mathematica [37], the low-frequency behaviour is readily obtained from the following limits:

$$
\lim _{u \longrightarrow 0} \frac{\Phi_{4}(u)}{\sqrt{u}}=\int_{0}^{\infty} \frac{\left[J_{1}(y)\right]^{2}}{y^{2}} d y=\frac{4}{3 \pi}
$$

and:

$$
\lim _{u \longrightarrow 0} \frac{\Phi_{5}(u)}{\sqrt{u}}=0
$$

in agreement with the previous derivation by Navarro-Laboulais et al. [8]. From Eqs. (A.1a), (A.2a) and (A.3a), we derive the diffusion resistance where the subscript 'FP' refers to Fleischmann and Pons:

$$
R_{\mathrm{d}, \mathrm{FP}}=\frac{16 R T}{3 n^{2} F^{2} \pi^{2} r_{\mathrm{e}} D c^{\mathrm{b}}}
$$

Next, the characteristic frequency corresponding to the apex of the diffusion impedance graph was given as $u_{\mathrm{c}, \mathrm{FP}}=2.5$ by Rotenberg et al. [40], while Navarro-Laboulais et al. [8] calculated numerically $u_{\mathrm{c}, \mathrm{FP}}=2.5119$. The more accurate value, $u_{\mathrm{c}, \mathrm{FP}}=2.440$, is proposed in this work [34].

Finally, minus the imaginary part of the diffusion impedance, calculated at the apex of the Nyquist diagram, satisfies $\left(\operatorname{Im} Z_{\mathrm{c}} / R_{\mathrm{d}}\right)_{\mathrm{FP}}=0.233[40]$, therefore:

$$
\operatorname{Im}\left(Z_{\mathrm{d}, \mathrm{FP}}\left(u_{\mathrm{c}}\right)\right)=-0.395 \frac{R T}{n^{2} F^{2} \pi r_{\mathrm{e}} D c^{\mathrm{b}}}
$$




\section{Figures}

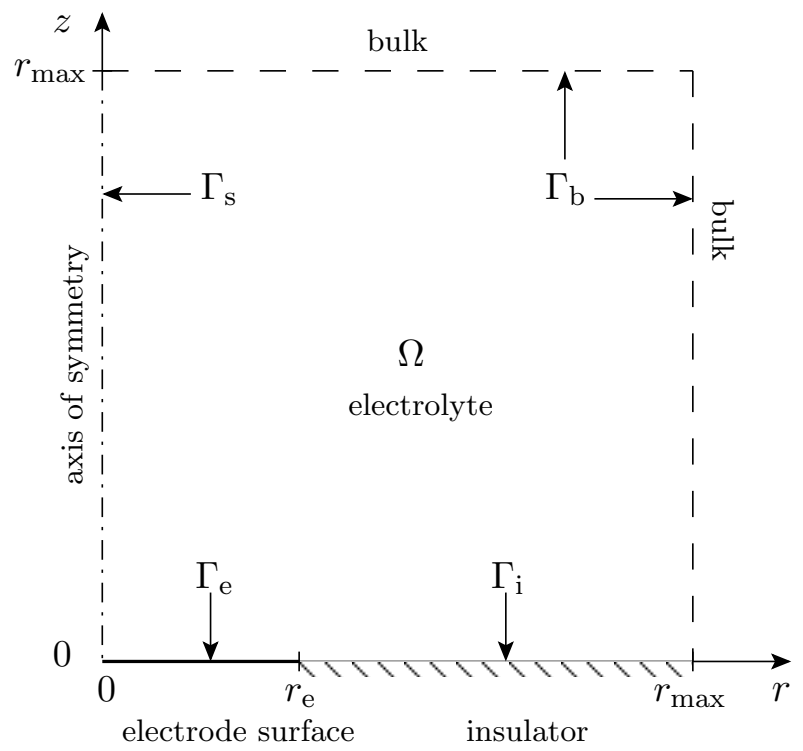

Figure 1 - Schematic representation of the finite element calculation domain, with cylindrical symmetry, and $r_{\max } / r_{\mathrm{e}}=4$, for a microdisk inlaid electrode. $\Omega$ : finite element calculation domain corresponding to a meridian subsection of the domain filled by the electrolytic solution; $\Gamma_{\mathrm{e}}$ : interface with the electronic conductor; $\Gamma_{\mathrm{s}}$ : axis of symmetry; $\Gamma_{\mathrm{i}}$ : interface with the insulator; $\Gamma_{\mathrm{b}}$ : electrolyte bulk.
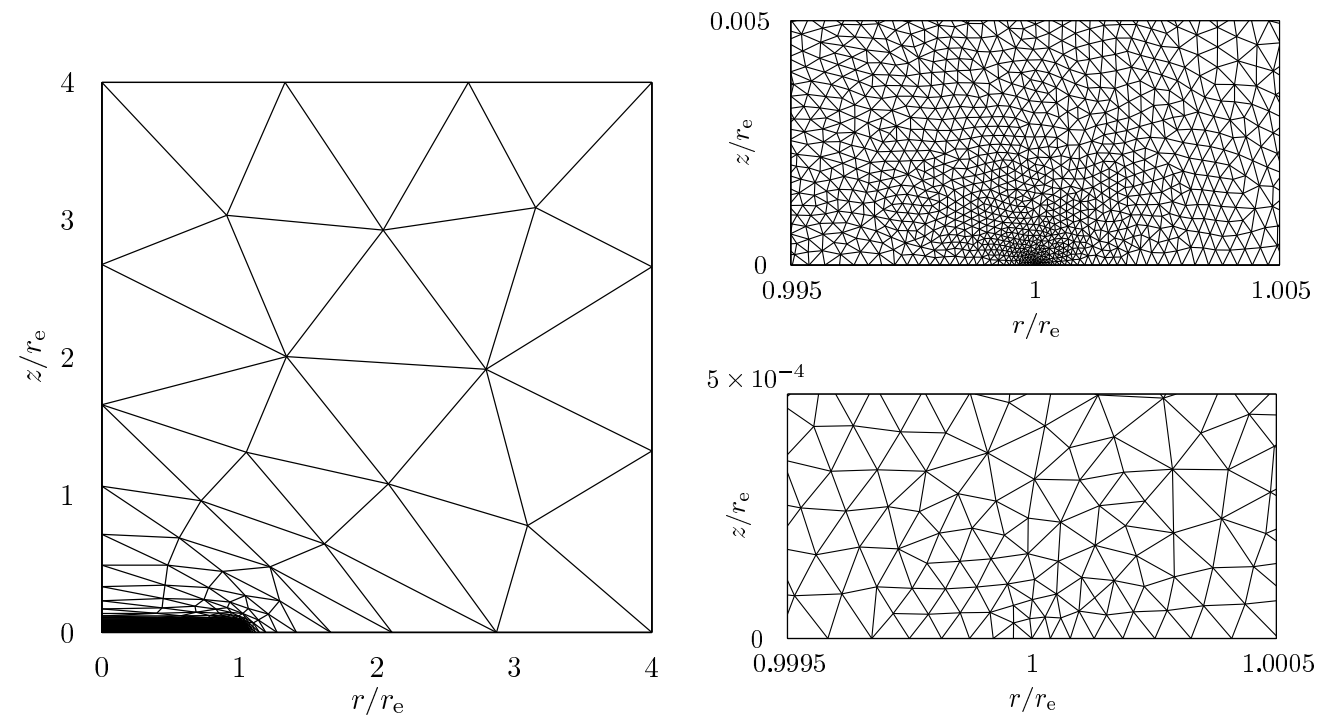

Figure 2 - Example of mesh generated by anisotropic adaptation algorithm in the high frequency range. Left: global view of the mesh corresponding to $r_{\max } / r_{\mathrm{e}}=4$ and presenting a boundary layer near the electronic conductor (the "dark zone"). Right: enlarged view in the neighbourhood of the singularity, with a magnification factor $400 \times 400$ (top) and $4000 \times 4000$ (bottom). 


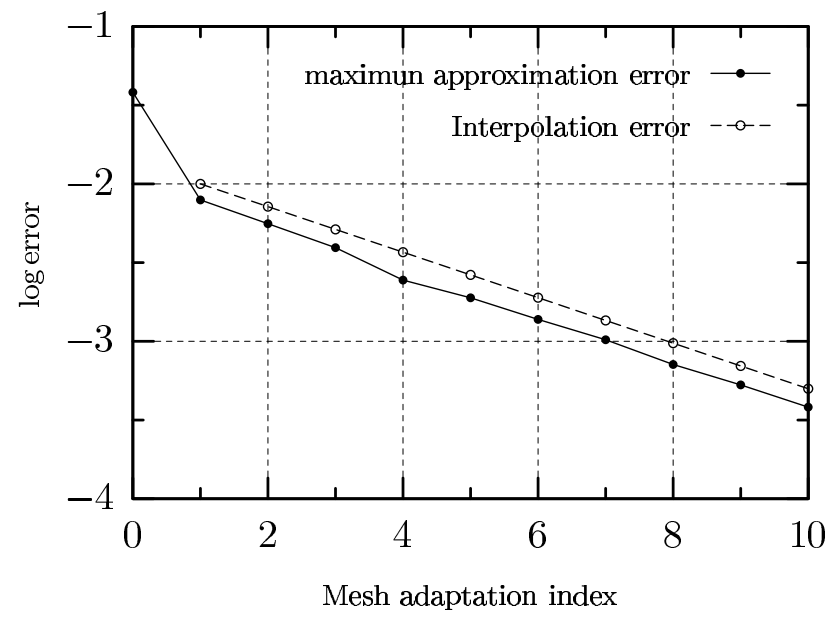

Figure 3 -Comparison between the imposed interpolation error and the resulting maximal approximation error on the steady-state concentration for a Nernstian system. The reference solution is taken from Crank and Furzeland [35]. Computation domain size $r_{\max } / r_{\mathrm{e}}=256$. Both errors are absolute dimensionless errors.
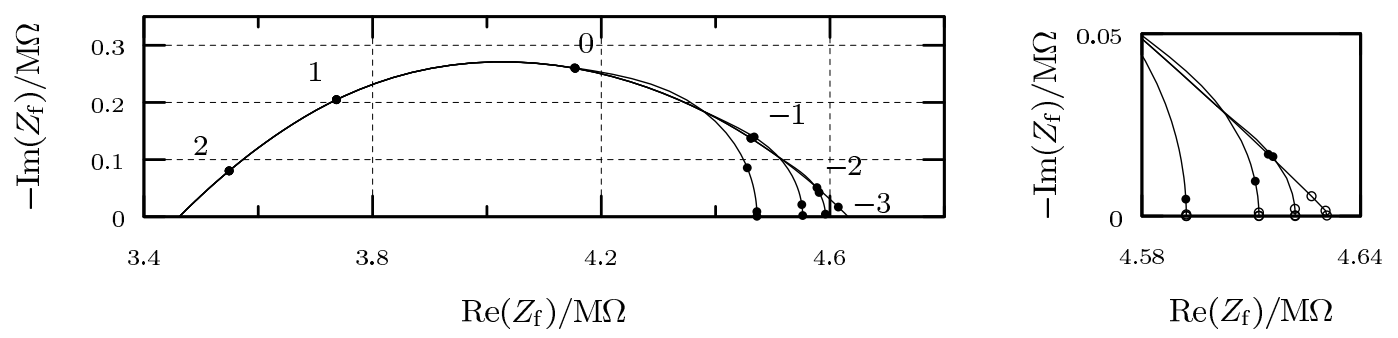

Figure 4 - Faradaic impedance relative to an inlaid microdisk electrode computed by adaptative FEM at the equilibrium potential for $n=1, k^{0}=10^{-3} \mathrm{~cm} \mathrm{~s}^{-1}, \alpha_{\mathrm{r}}=\alpha_{\mathrm{o}}=1 / 2, D_{\mathrm{R}}=D_{\mathrm{O}}=$ $5 \times 10^{-6} \mathrm{~cm}^{2} \mathrm{~s}^{-1}, c_{\mathrm{R}}^{\mathrm{b}}=c_{\mathrm{O}}^{\mathrm{b}}=10^{-5} \mathrm{~mol} \mathrm{~cm}{ }^{-3}, r_{\mathrm{e}}=10^{-3} \mathrm{~cm}$ and $T=298 \mathrm{~K}$. Left: Nyquist plot, for $r_{\max } / r_{\mathrm{e}}=4,8,16,512$ from left to right in the low-frequency domain. Some values of the decimal logarithm of $f / \mathrm{Hz}$ are reported on the graphs. Right: enlarged view of the impedance diagrams in the low-frequency domain for $r_{\max } / r_{\mathrm{e}}=16,32,64,512$; full circles correspond to $f=10^{-3} \mathrm{~Hz}$ and empty circles correspond to the values $-4,-5$ and -6 of the decimal logarithm of $f / \mathrm{Hz}$ from top to bottom. 


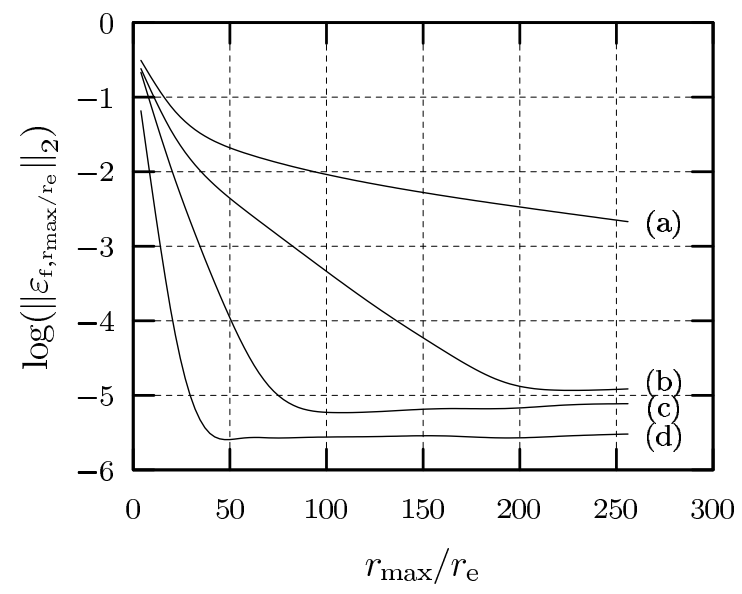

Figure 5 - Evolution of the decimal logarithm of the error norm $\left\|\varepsilon_{\mathrm{f}, \mathrm{r} \max / \mathrm{r} e}\right\|_{2}$ in Eq. (24) for the Faradaic impedance convergence with respect to the size $r_{\max } / r_{\mathrm{e}}$ of the computational domain. The Faradaic impedance is computed at the equilibrium potential of the microdisk electrode and its convergence error is evaluated for $f / \mathrm{Hz} \geq 0$ (a), $f / \mathrm{Hz} \geq 10^{-4}$ (b), $f / \mathrm{Hz} \geq 10^{-3}$ (c) and $f / \mathrm{Hz} \geq 10^{-2}(\mathrm{~d})$. The values of parameters are the same as in Fig. 4, so the corresponding dimensionless frequency $u=2 \pi f r_{\mathrm{e}}^{2} / D_{\mathrm{R}}$ is such that $u \geq 0$ (a), $u \geq 1.25 \times 10^{-4}$ (b), $u \geq$ $1.25 \times 10^{-3}$ (c) and $u \geq 1.25 \times 10^{-2}(\mathrm{~d})$.

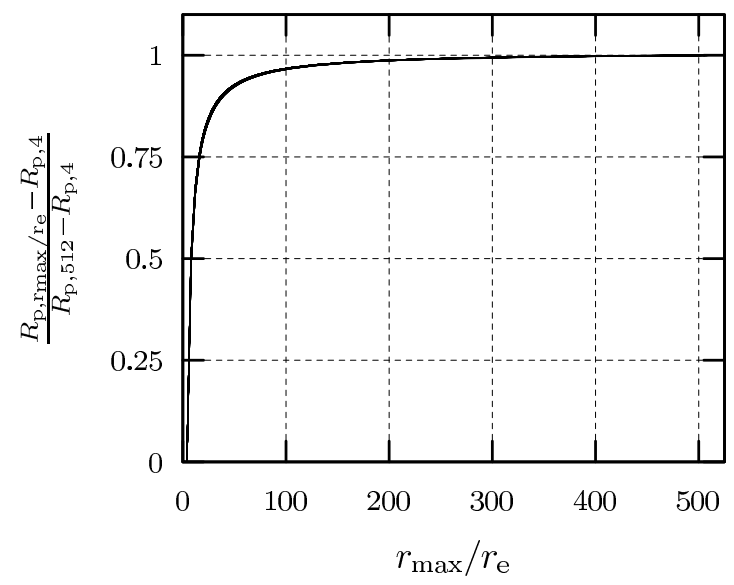

Figure $6-$ Normalized error for computation of the polarization resistance $\left(R_{\mathrm{p}, \mathrm{r}_{\max } / \mathrm{r}_{\mathrm{e}}}-R_{\mathrm{p}, 4}\right) /\left(R_{\mathrm{p}, 512}-R_{\mathrm{p}, 4}\right)$ plotted with respect to the size $r_{\max } / r_{\mathrm{e}}$ of computational domain. All the curves plotted for a standard rate constant of electron transfer $k^{0} / \mathrm{cm} \mathrm{s}^{-1}$ varying from $10^{-5}$ to 10 are superposed. The other parameters have the same values as in Fig 4 . 

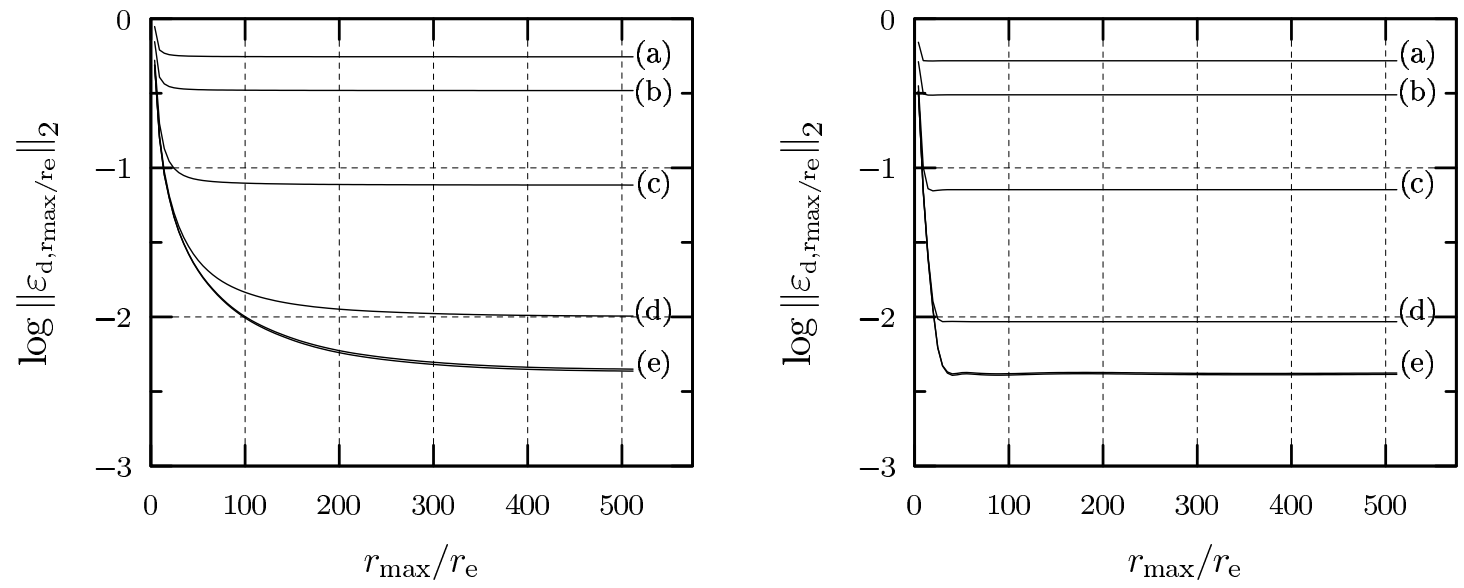

Figure 7 - Comparison of the diffusion impedance $Z_{\mathrm{d}, \mathrm{r}_{\max } / \mathrm{r}_{\mathrm{e}}}(u)$, computed by FEM, with $Z_{\mathrm{d}, \mathrm{FP}}(u)$ predicted from Fleischmann and Pons, depending on the size $r_{\max } / r_{\mathrm{e}}$ of the computational domain, using the relative deviation norm in Eq. (25). The relative deviation is calculated for $f / \mathrm{Hz} \geq 0$ (left) and for $f / \mathrm{Hz} \geq 10^{-3}$ (right). In both cases, $k^{0} / \mathrm{cm} \mathrm{s}^{-1}$ is equal to $10^{-1}$ (a), $10^{-2}$ (b), $10^{-3}$ (c), $10^{-4}$ (d), $10^{-5}$ and $10^{-6}$ (e) and the diffusion impedances are calculated at the equilibrium potential of the microdisk electrode. The other parameters have the same values as in Fig. 4.
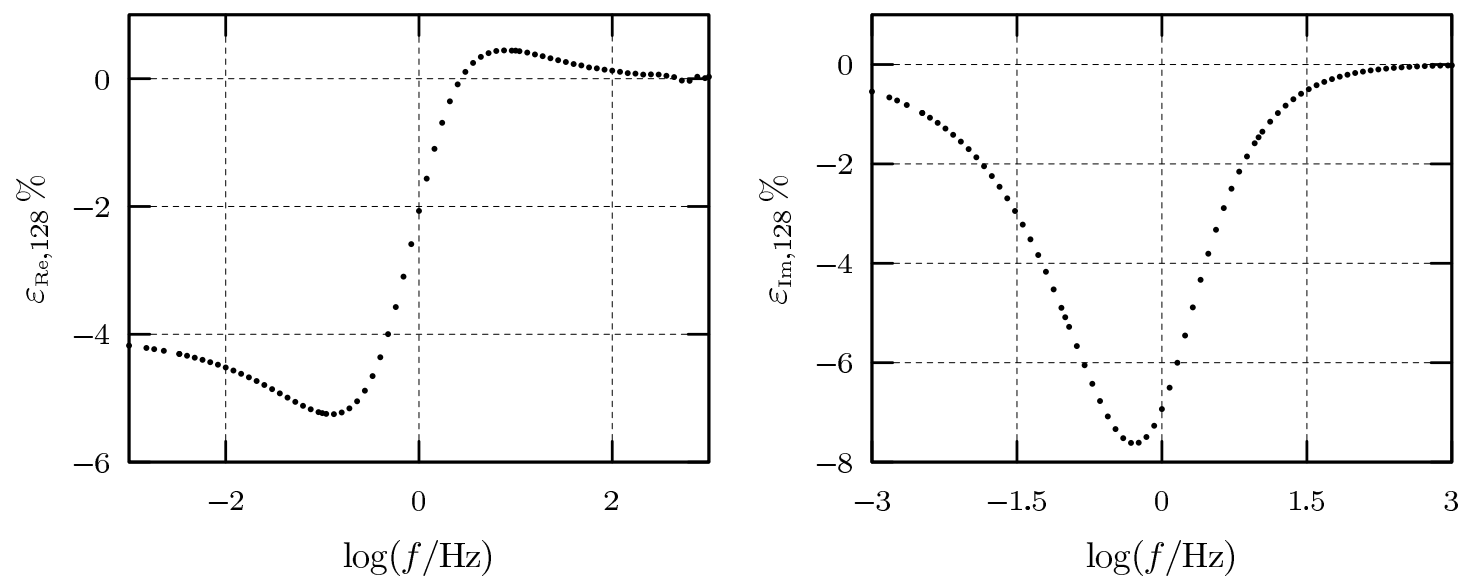

Figure 8 - Comparison of the diffusion impedance $Z_{\mathrm{d}, 128}(u)$, computed by FEM for $r_{\max } / r_{\mathrm{e}}=$ 128 , with $Z_{\mathrm{d}, \mathrm{FP}}(u)$ predicted from Fleischmann and Pons formulae, as a function of the decimal logarithm of frequency $/ \mathrm{Hz} . k^{0} / \mathrm{cm} \mathrm{s}^{-1}=10^{-2}$. The other parameters have the same values as in Fig. 4. Left: relative deviation of the real part. Right: relative deviation of the imaginary part. 

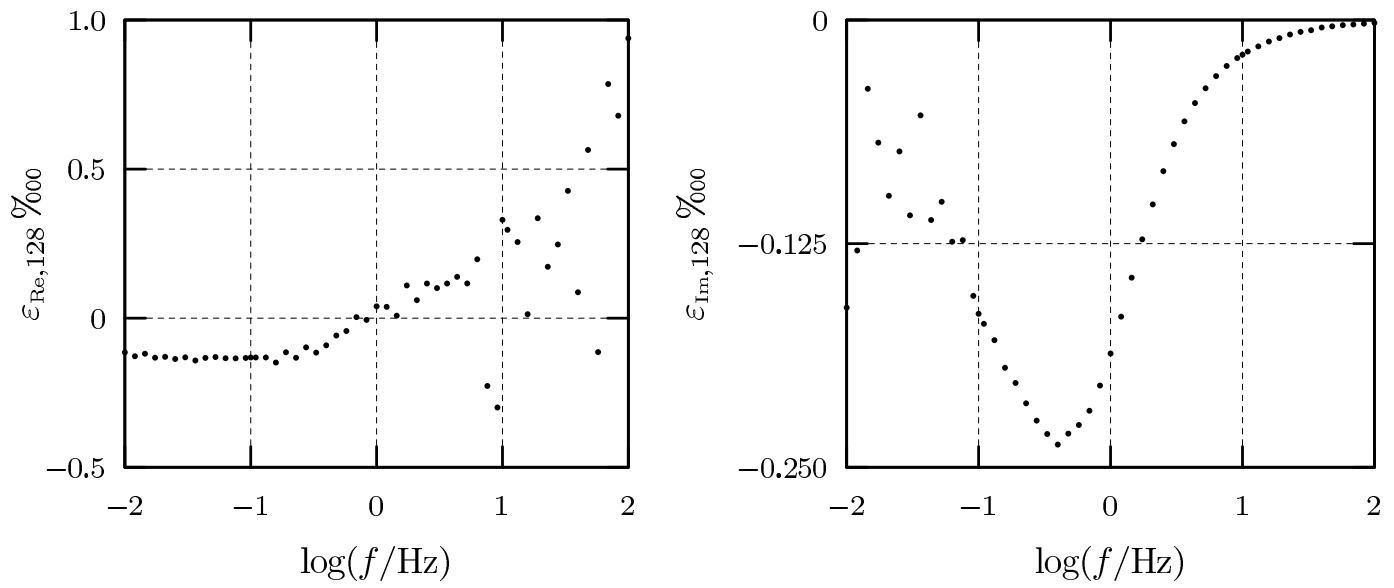

Figure 9 - Same figure caption as in Fig. 8, except for $k^{0} / \mathrm{cm} \mathrm{s}^{-1}=10^{-6}$. Scattering of numerical data is due to the computational noise which is of the same order of magnitude as the relative deviation in \%oo.
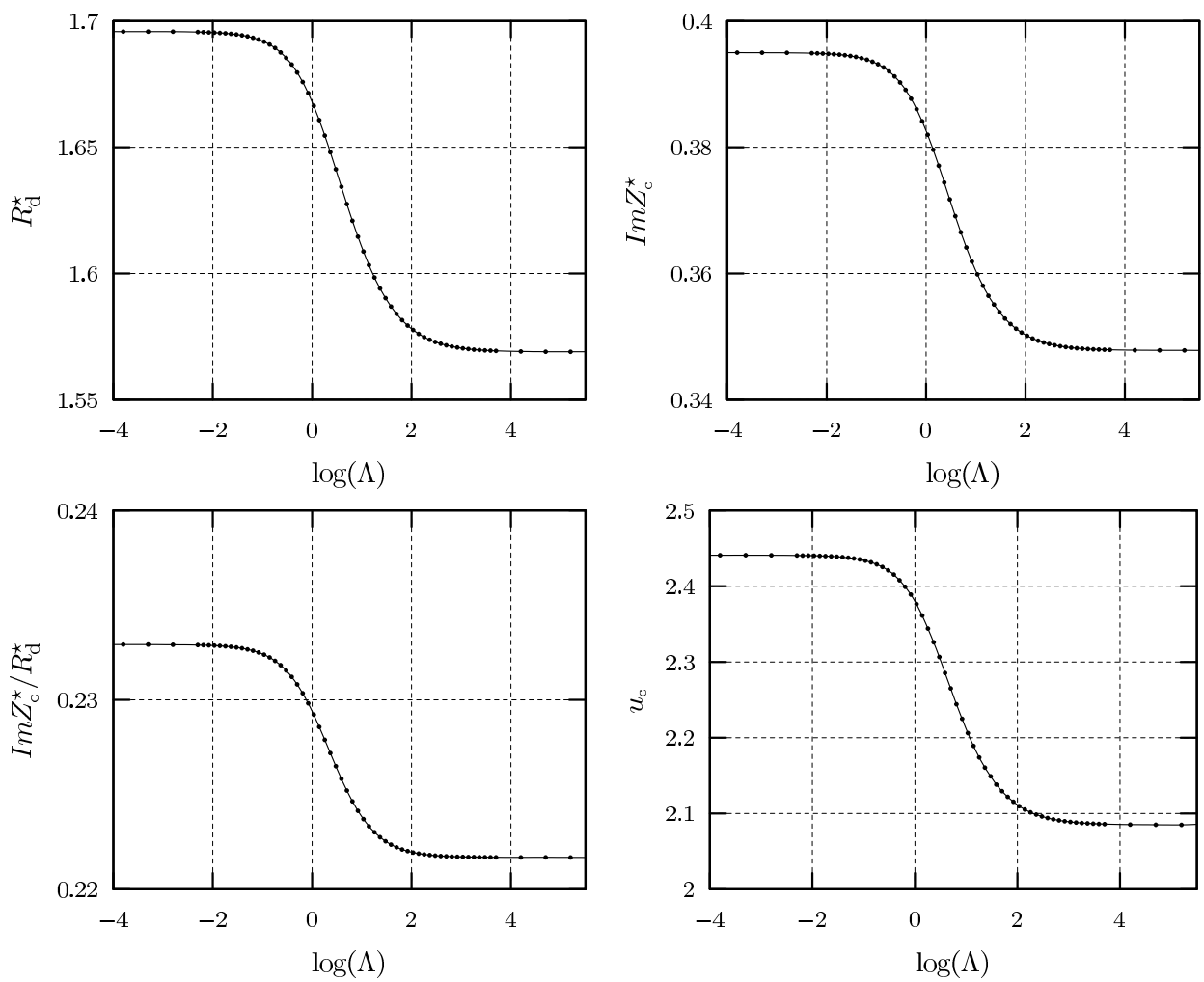

Figure 10 - Evolution of the characteristic quantities of the diffusion impedance graph computed by FEM vs $\log \Lambda$, with $\Lambda=k^{0} r_{\mathrm{e}} / D_{\mathrm{R}}$. The numerical data (dots) have been fitted (solid lines) using Eq. (29). The best-fitted values of parameters have been reported in Tab. 1. 


\section{Table}

\begin{tabular}{ccccccc}
\hline $\begin{array}{c}\text { Characteristic } \\
\text { quantity }\end{array}$ & $a_{1}$ & $a_{2}$ & $b_{1}$ & $b_{2}$ & $b_{3}$ & $\chi^{2}$ \\
\hline$R_{\mathrm{d}}^{\star}$ & 1.6957 & 0.1268 & 1.8968 & 1.7836 & 1.4239 & $5.7 \times 10^{-7}$ \\
$u_{\mathrm{c}}$ & 2.4410 & 0.3560 & 2.4656 & 1.9235 & 1.4205 & $3.2 \times 10^{-5}$ \\
$\operatorname{Im} Z_{\mathrm{c}}^{\star}$ & 0.3950 & 0.0472 & 1.7013 & 1.8818 & 1.3467 & $1.3 \times 10^{-7}$ \\
\hline
\end{tabular}

Table 1 - Best-fits of the numerical coefficients in Eq. (29) relative to $R_{\mathrm{d}}^{\star}, \operatorname{Im} Z_{\mathrm{c}}^{\star}$ and $u_{\mathrm{c}}$ which can be used to predict the diffusion resistance at an inlaid microdisk electrode under the same operating conditions as in Ref. [7], as well as the characteristic frequency at the apex of the Nyquist impedance diagram, and finally the imaginary part of the diffusion impedance at this apex, from Eqs. (26)-(28), respectively. The best values have been obtained by nonlinear fitting of the numerical data in Fig. 10. 